\title{
LA-UR-14-28102
}

Approved for public release; distribution is unlimited.

Title: $\quad$ Direct multiscale embedding of viscoplastic self-consistent (VPSC) polycrystal plasticity enabled by adaptive sampling

Author(s): $\quad$ Barton, Nathan

Lebensohn, Ricardo A.

Intended for: Web

Issued:

2014-10-16 
Disclaimer:

Los Alamos National Laboratory, an affirmative action/equal opportunity employer,is operated by the Los Alamos National Security, LLC for the National NuclearSecurity Administration of the U.S. Department of Energy under contract DE-AC52-06NA25396. By approving this article, the publisher recognizes that the U.S. Government retains nonexclusive, royalty-free license to publish or reproduce the published form of this contribution, or to allow others to do so, for U.S. Government purposes. Los Alamos National Laboratory requests that the publisher identify this article as work performed under the auspices of the U.S. Departmentof Energy. Los Alamos National Laboratory strongly supports academic freedom and a researcher's right to publish; as an institution, however, the Laboratory does not endorse the viewpoint of a publication or guarantee its technical correctness. 


\section{Direct multiscale embedding of viscoplastic self-consistent (VPSC) polycrystal plasticity enabled by adaptive sampling}

Nathan Barton (LLNL) and Ricardo Lebensohn (LANL) 


\section{Adaptive Sampling builds response on the fly}

- Coarse scale model queries database for finescale material response

- If possible, approximate response from past evaluations

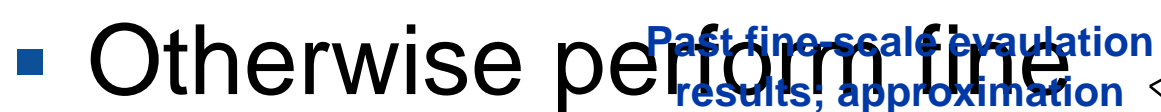
scale evaluation

- Fine-scale evaluations grow database

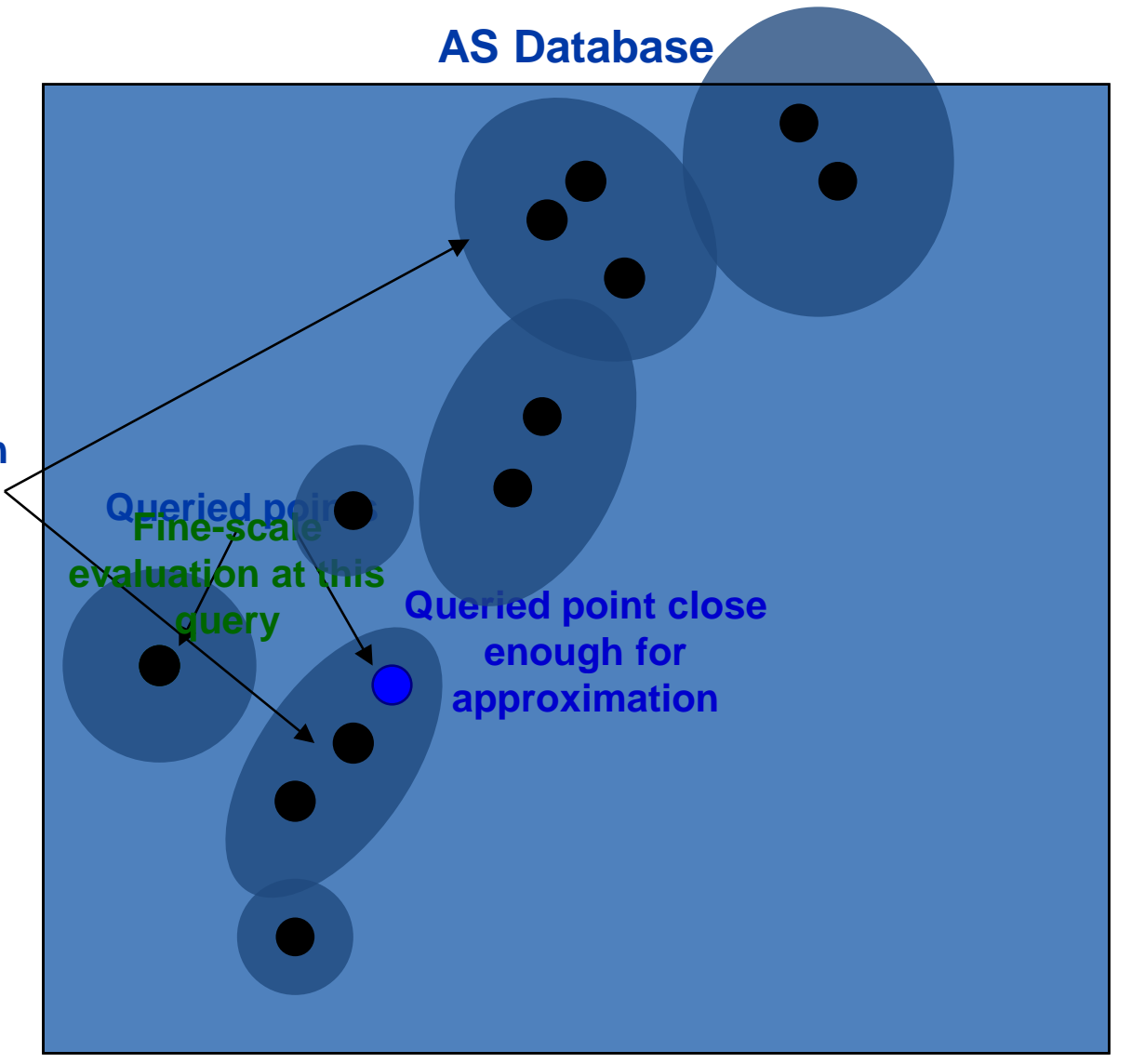




\section{Speedup and error control are well behaved}

- 250X speedup: drawing, Taylor, with 512 grains at the fine-scale

- Full evaluation simulation: roughly 2.3 processor-years
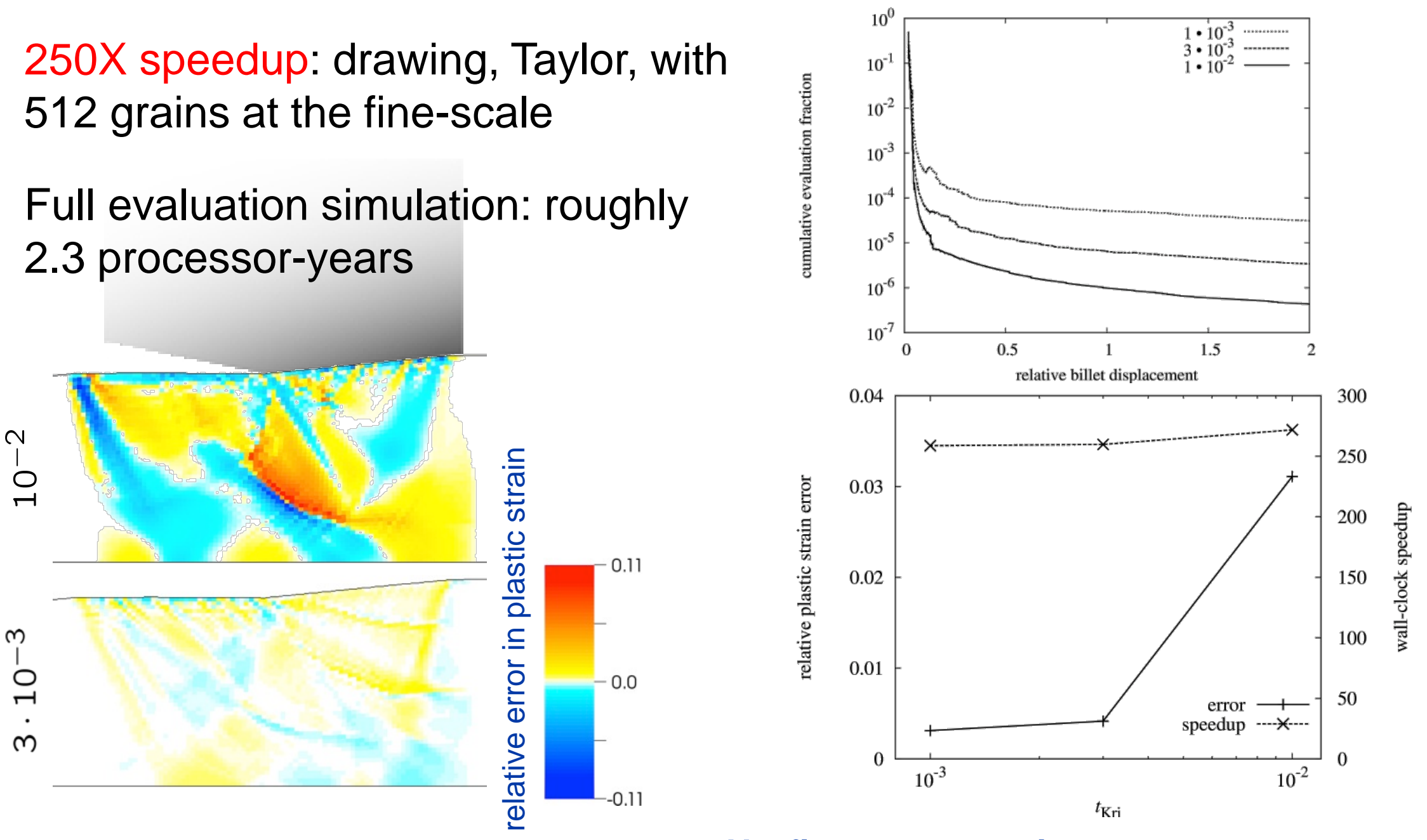

AS speedup $\approx \frac{N W}{n W+N w}$

- $N$ : fine-scale queries

- $n$ : fine-scale evaluations under AS

- $W$ : fine-scale work

- w: AS work in lieu of fine-scale evaluation 


\section{Low symmetry and low-SFE cubic single crystals often twin, substantially affecting polycrystal response}

HCP Metals: $\mathrm{Zr}$ (Nuclear), Ti (Aero), Mg (Auto), Be (defense): Slip:
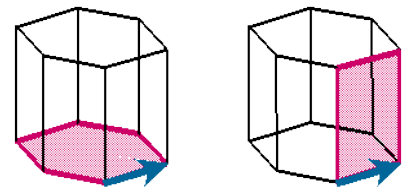

$(0001)\langle 11 \overline{2} 0\rangle$

Basal

slip

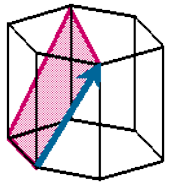

$(11 \overline{2} 2)(11 \overline{2} 3$

Pyramidal slip
Twinning:

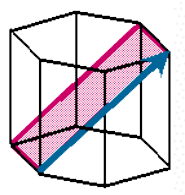

$(10 \overline{1} 2)(10 \overline{1} 1$

Tensile twin

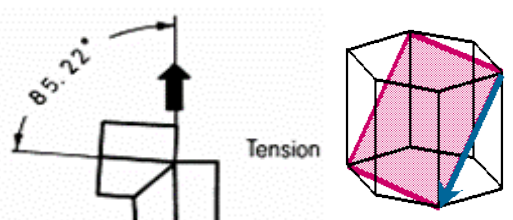

$(10 \overline{1} 1)(10 \overline{1} 2\rangle$

Compressive twin
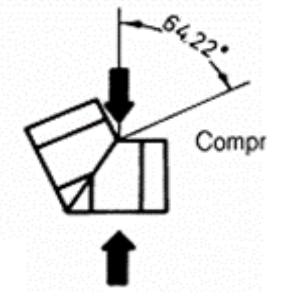

$\{112 \overline{2} 2\}<\overline{11} 23>$ $(1122)<1123>$
$s=0.225$
Rapid texture changes, anisotropic hardening:

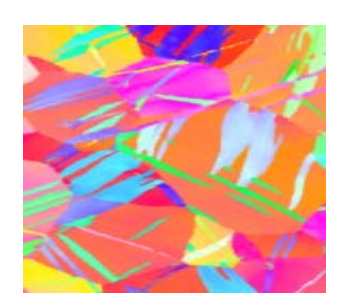

Strong anisotropy, tension-

Twinning:
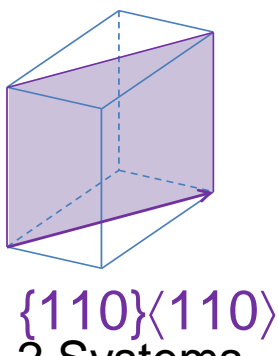
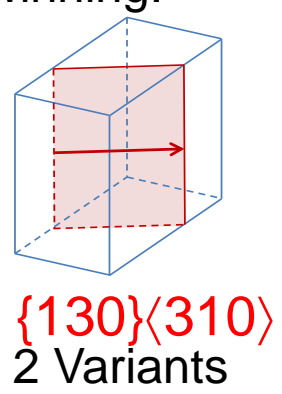

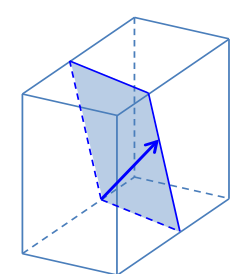

' $\{172\} '\langle 312\rangle$

4 Variants

$(010)[100]$
1 System (001) 1001
1 System 2 Systems

FCC, BCC, low SFE, high strain-rate:

Slip:

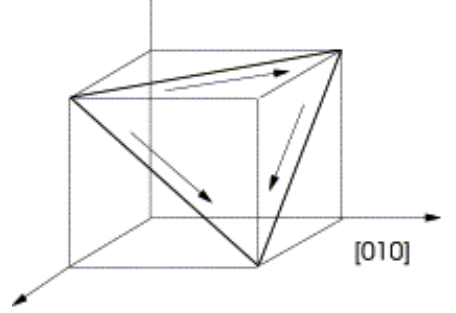

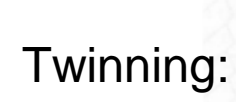

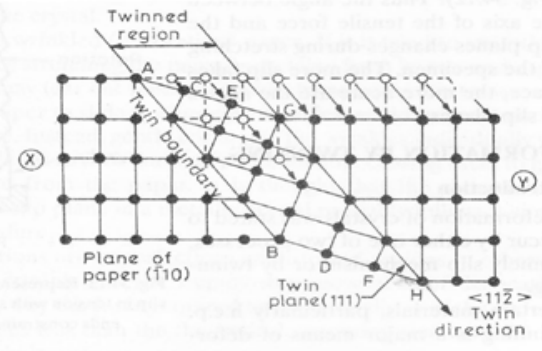

compression asymmetry:
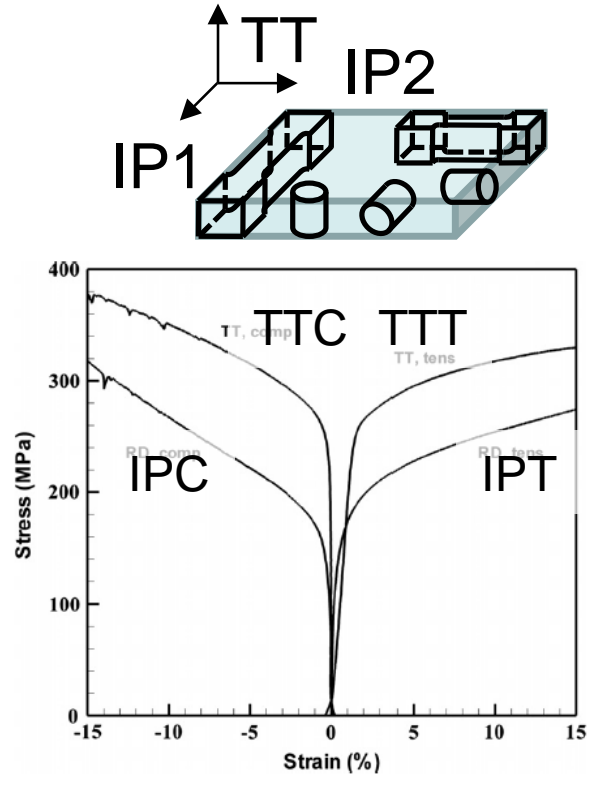


\section{ViscoPlastic Self-Consistent (VPSC) formulation}

Lebensohn and Tome, Acta Mater (1993)

- VPSC model:

- VPSC vs Taylor:

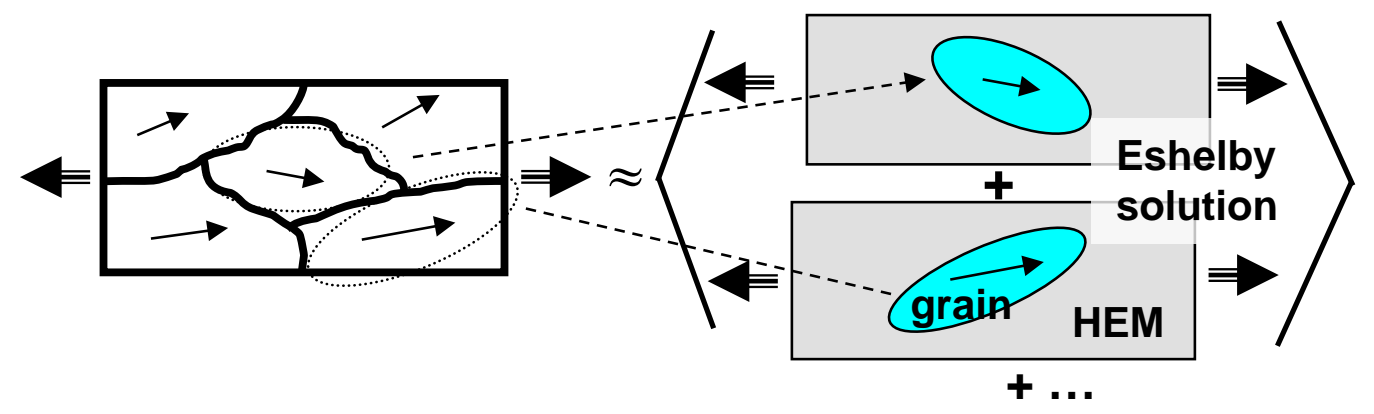

Unlike under Taylor model, each grain deform differently according to its directional properties.

- VPSC code:

Distribution version: widely used in Universities, Laboratories and Industry.

- VPSC-based Abaqus UMAT:

Available (Segurado, Lebensohn, Tome, IJP, 2012), based on "full evaluation".

- Material systems studied with VPSC:

Metals: Al-alloys, Cu-alloys, Zr-alloys, Ti-alloys, Mg-alloys, Be, Ni-alloys, steels, U-alloys, etc. Geomaterials: ice, calcite, quartzite, halite, olivine, etc. Polymers: Polyethylene 


\section{VPSC-Abaqus prediction of bending of rolled Ti bars}

Eglin's Ti plate, orthotropic texture (*):

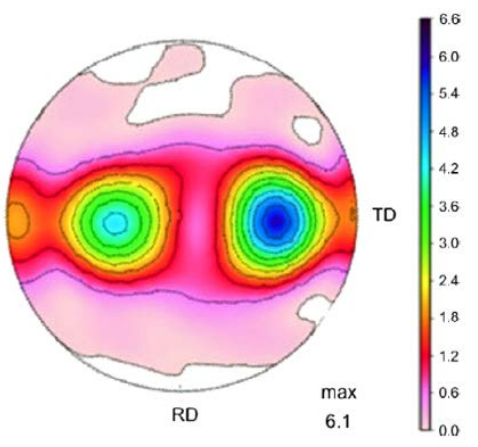

4-point bending jig:

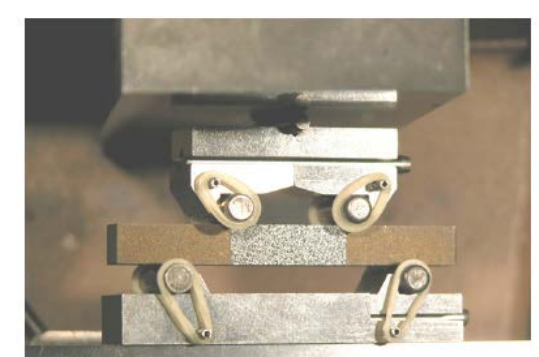

4 different configurations:

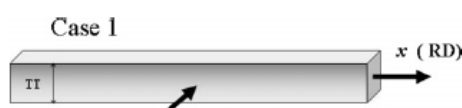

$y$ (TD)

Case $2^{z(\mathrm{TT})}$ Loading Direction

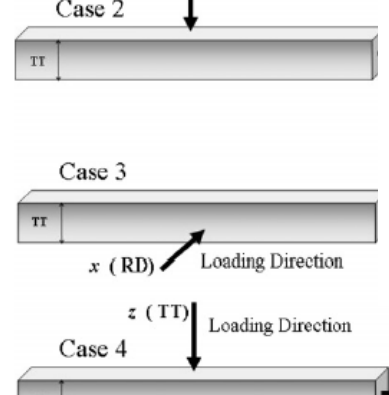

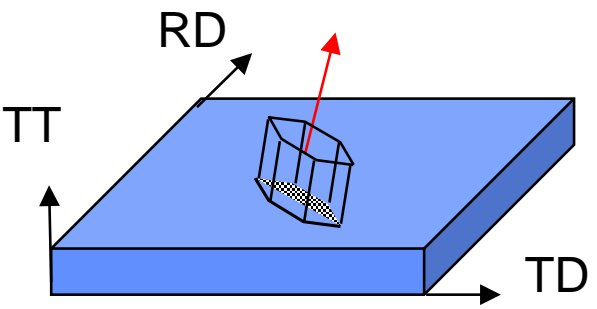

Animated simulation:

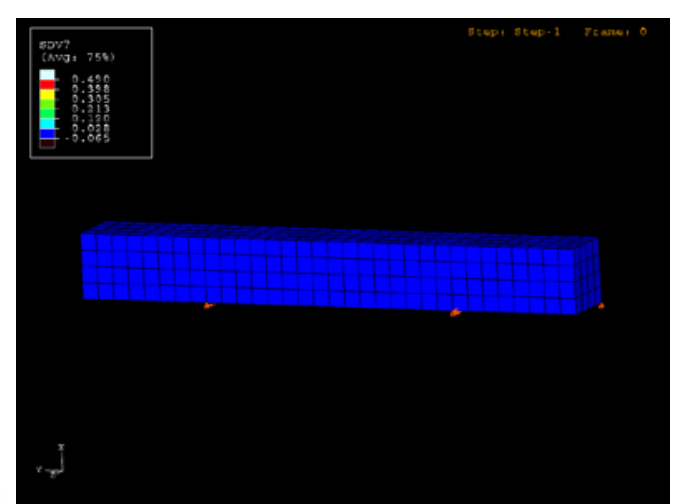

Different final 3-D shapes:

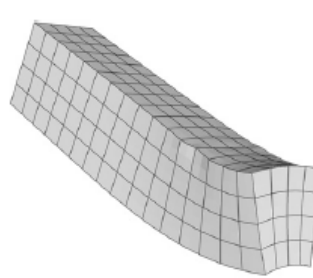

TT vertical

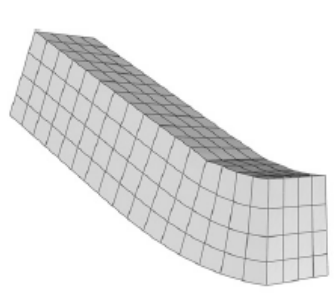

TT horizontal

(*) Nixon et al. IJP, 2010; (**) Knezevic et al, MSE A, 2013

Uniaxial tests: anisotropic, tension $\neq$ compres (twinning!) (*):
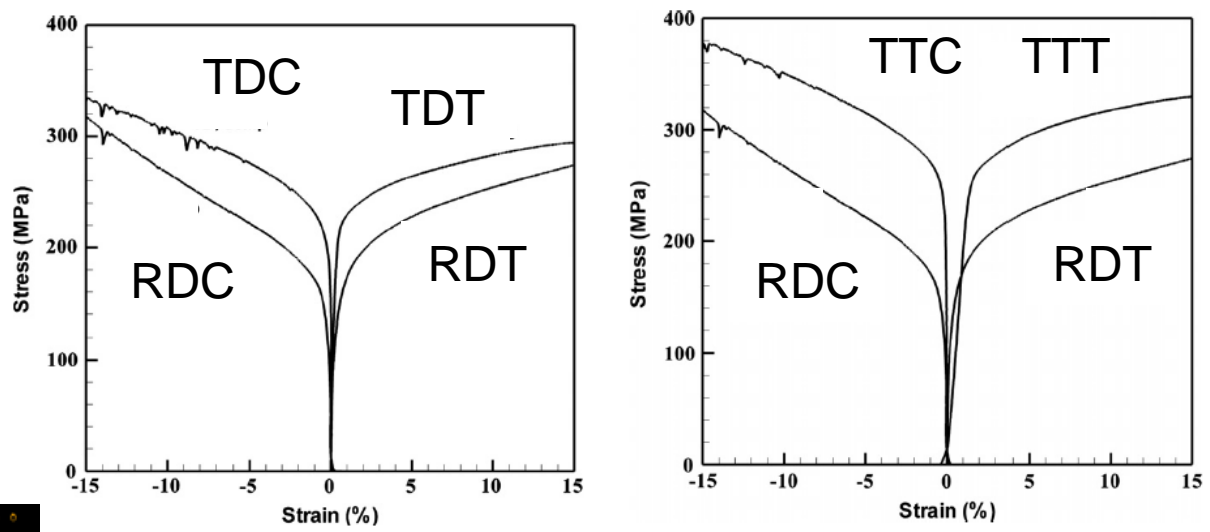

VPSC-Abaqus (green symbols) vs experimental cross-sections (photographs) $(* *)$ :
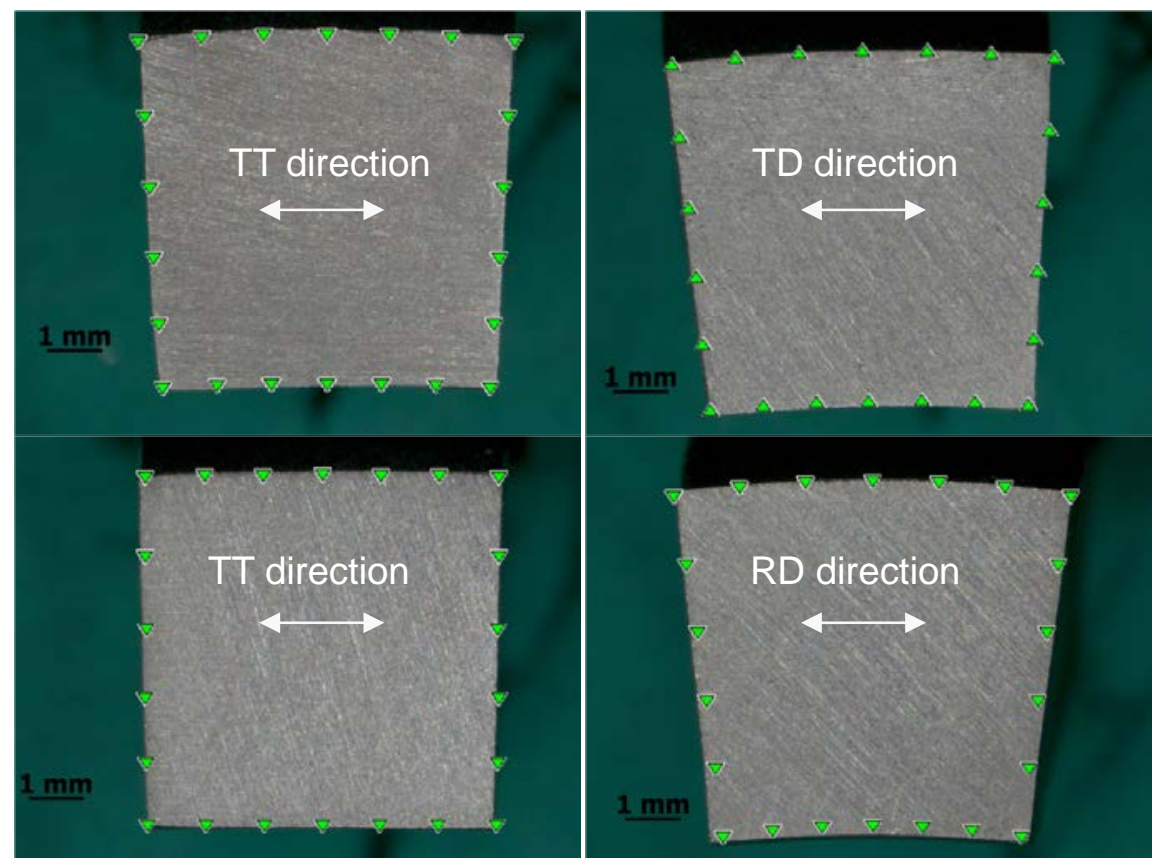


\section{Previous AS implementation (Taylor) employed a simple fine-scale state description}
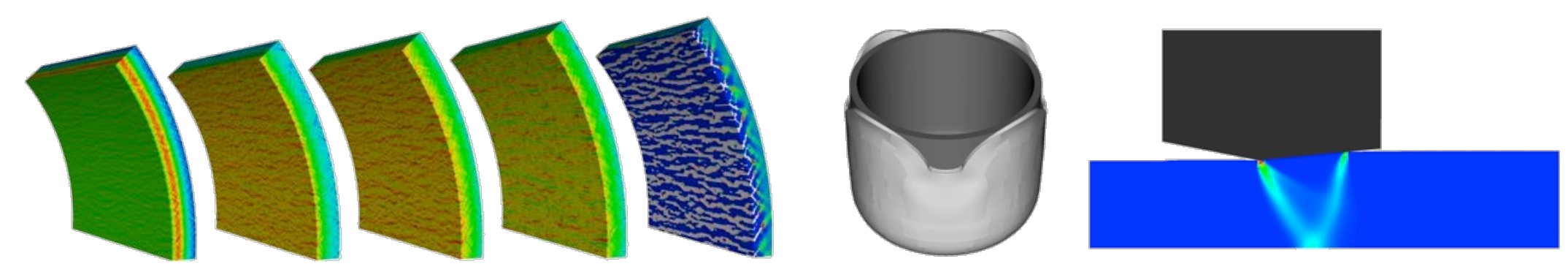

- Scalar hardness variable(s) $\mathcal{H}=\left\{h_{1}, h_{2}\right\}$

- no texture variation or evolution beyond rigid rotation

- $\approx 10$ dimensional sampling space

- adequate for initial anisotropy, strain localization, pressure and temperature sensitivity

AS-VPSC makes use of a more expansive fine-scale state description

$$
\mathcal{H}=\left\{h_{1}, h_{2}, h_{3}, \ldots, A_{i j}, c_{k}\right\}
$$




\section{Texture (ODF) evolution follows from standard conservation equation, and allows for twinning}

Conservation equation: $\quad \frac{\partial \rho}{\partial t}+\operatorname{div}(\rho \mathbf{v})=\Theta$

Weak form:

$$
\begin{array}{r}
\int_{\mathcal{R}}\left(\frac{\partial \rho}{\partial t} \phi+\phi \operatorname{div}(\rho \mathbf{v})\right) \mathrm{d} \mu=\int_{\mathcal{R}}\left(\frac{\partial \rho}{\partial t} \phi-\right. \\
\frac{\partial \rho}{\partial t} \sim\left(\rho_{n+1}-\rho_{n}\right) / \Delta t
\end{array}
$$

Forward-Euler integration:

$$
\int_{\mathcal{R}} \rho_{n+1} \phi \mathrm{d} \mu=\int_{\mathcal{R}}\left(\rho_{n} \phi+(\Delta t)\left(\rho_{n} \mathbf{v} \cdot \nabla \phi+\Theta_{n} \phi\right)\right) \mathrm{d} \mu
$$

Spatial discretization, no twinning: $\quad \bar{\rho}_{n+1}=\bar{\rho}_{n}+L^{-1} G\left(\bar{\rho}_{n} \bar{V}\right) \Delta t$

Twinning operators constructed similarly: $\quad \bar{f} \cdot\left(L_{\mathrm{t}} \bar{g}\right)=\int_{\mathcal{R}} f(\mathbf{r}) g\left(\mathbf{r r}_{\mathrm{t}}\right) \mathrm{d} \mu$

Or using orthonormal basis functions: $\quad \rho=\sum_{i} c_{i} H_{i} \quad c_{i}=\int_{\mathcal{R}} \rho H_{i} \mathrm{~d} \mu$

$$
\frac{\mathrm{d} c_{i}}{\mathrm{~d} t}=\int_{\mathcal{R}} \frac{\partial \rho}{\partial t} H_{i} \mathrm{~d} \mu=\int_{\mathcal{R}}(\Theta-\operatorname{div}(\rho \mathbf{v})) H_{i} \mathrm{~d} \mu=\int_{\mathcal{R}} \rho \mathbf{v} \cdot \nabla H_{i} \mathrm{~d} \mu+\int_{\mathcal{R}} \Theta H_{i} \mathrm{~d} \mu
$$




\section{Pre-computation is unfeasible:}

\section{Even reducing to a small (coarse) harmonic basis, computation time is daunting}

- VPSC calculation takes $\approx 1$ second

- Evaluation results depend on current texture, so need to span space of harmonic basis and deformation conditions ... say $8^{60}$ evaluations, or $10^{46}$ processor-years

$\mathcal{Y}=f_{\mathcal{Y}}\left(\overline{\boldsymbol{\tau}}^{\prime}, \mathcal{H}, p, \theta\right)$

$$
\mathcal{H}=\left\{h_{1}, h_{2}, h_{3}, \ldots, A_{i j}, c_{k}, v_{\mathrm{tw}}\right\}
$$

- But a given simulation samples a comparatively lowdimensional manifold in this high-dimensional space! 


\section{Discrete harmonics offer an}

\section{appealing basis}

Spherical harmonics are eigenfunctions of the negative Laplacian on the $n$-sphere:

$$
-\Delta u=\lambda u
$$

Weak form:

$$
\int \nabla u \nabla \phi=\lambda \int u \phi, \forall \phi
$$

Discrete harmonics satisfy the generalized eigenvalue problem:

$$
H \bar{u}=\lambda L \bar{u}
$$
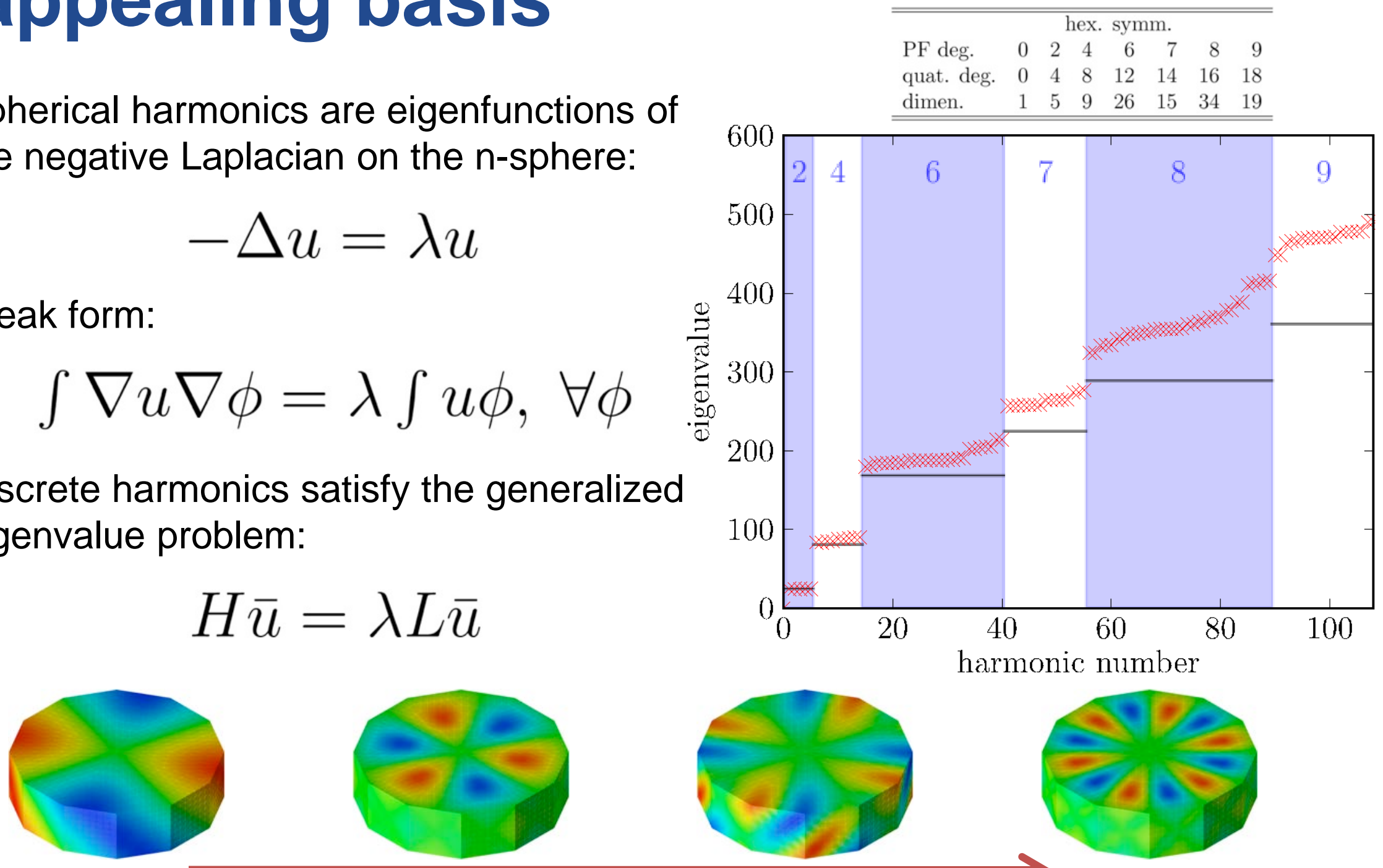


\section{Twinning operators amount to non- local source/sink relationships}

- Twinning operators involve the construction of modified 2-form "mass" ( $L-2$ inner product) matrices:

$$
\bar{f} \cdot\left(L_{\mathrm{t}} \bar{g}\right)=\int_{\mathcal{R}} f(\mathbf{r}) g\left(\mathbf{r r}_{\mathrm{t}}\right) \mathrm{d} \mu
$$

- Example: image of Gaussian at the origin under six twinning operators

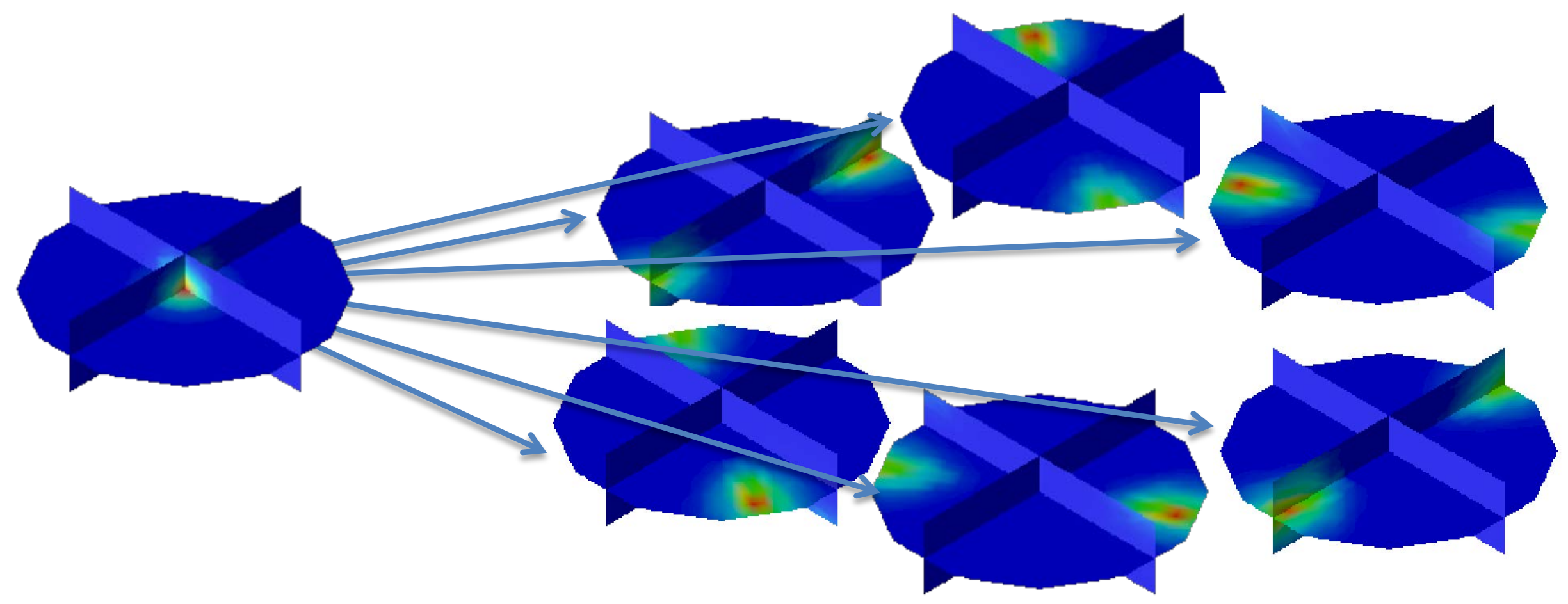




\section{Uniaxial deformation to $25 \%$ strain illustrates convergence}

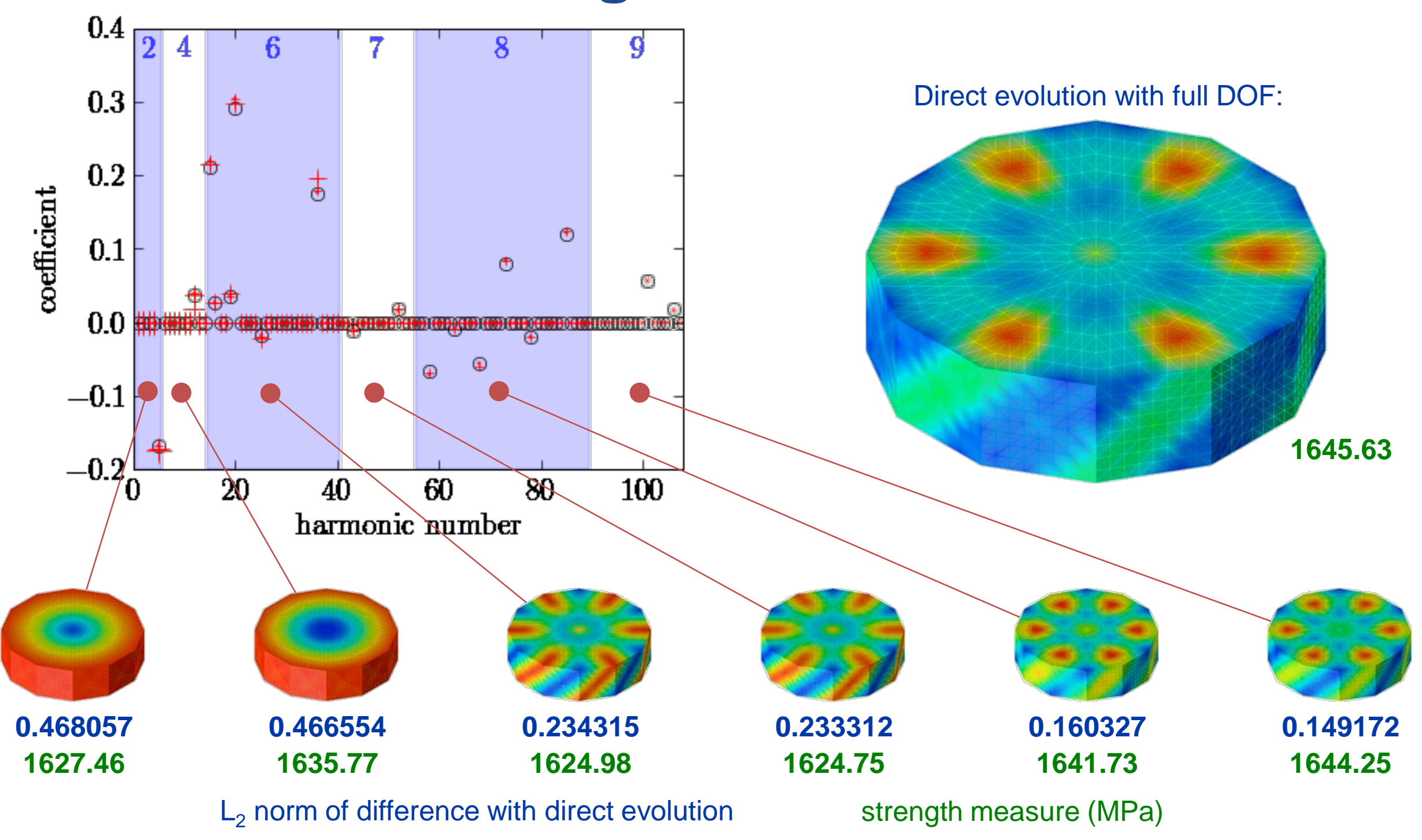




\section{Discrete aggregate results agree with direct continuous evolution methods}
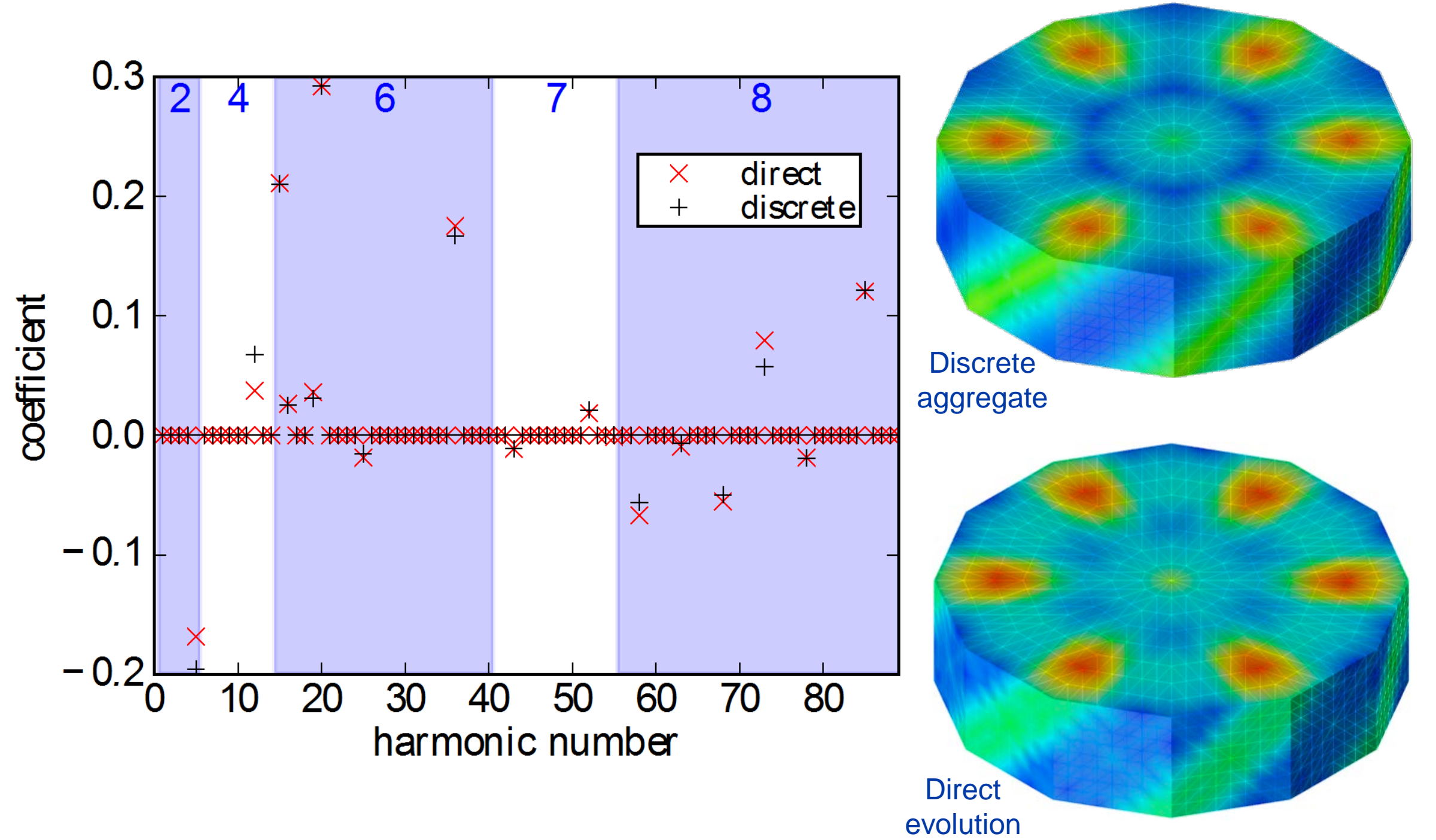


\section{Even under monotonic loading, sampling provides benefit}

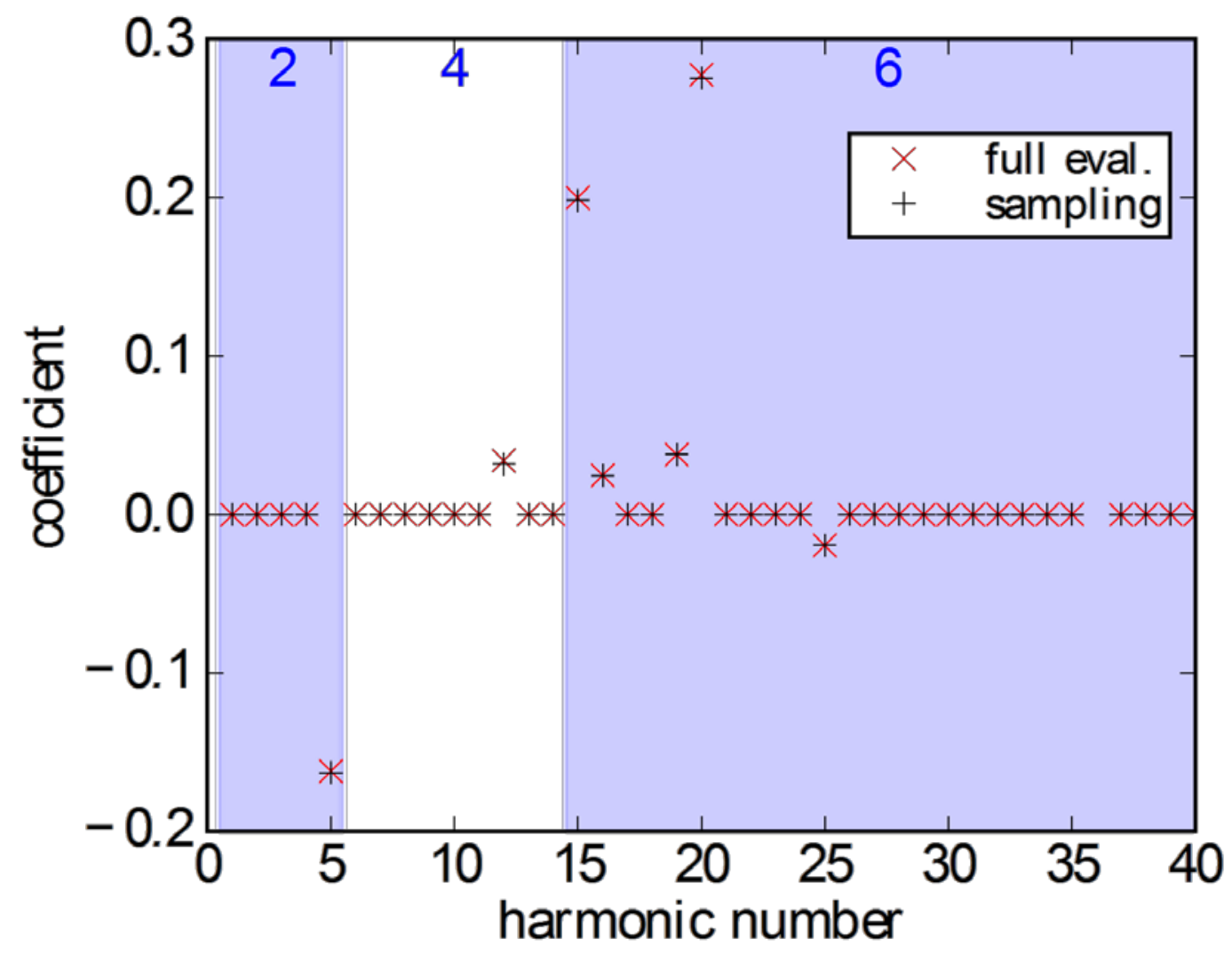

Evaluation fraction $<0.1$ 


\section{Example application - four-point bend of textured titanium}

- Initial texture from pole figures

- Project onto harmonics to get initial coefficients
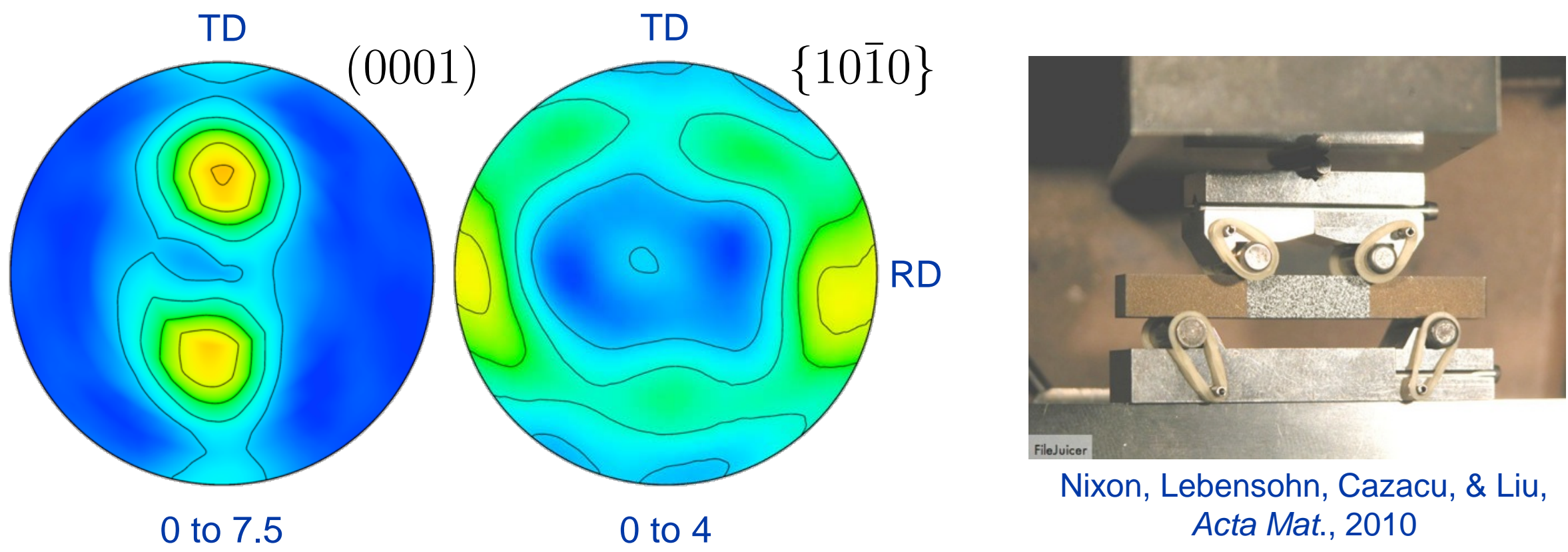


\section{Harmonic-based scheme captures salient features of the response}

- Compared to (points) PTR scheme with many more degrees of freedom \& distinct hardening in each grain

- Do not expect complete agreement - models employ different assumptions
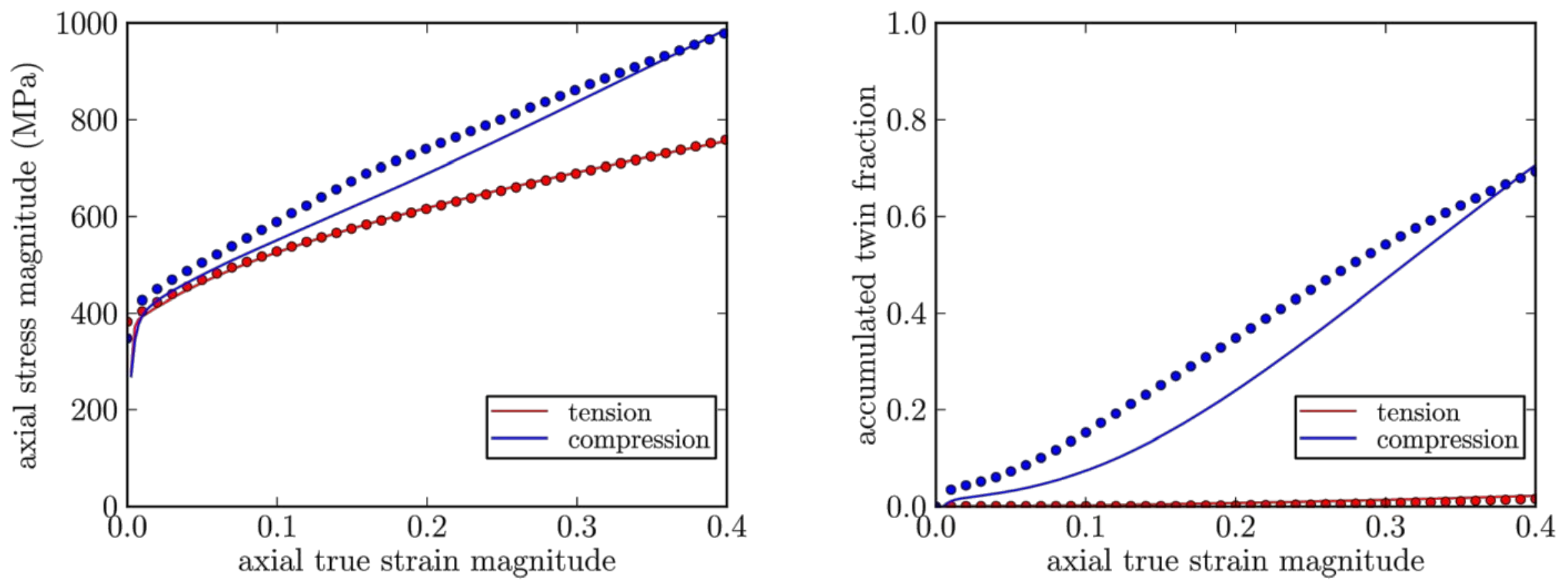


\section{Four-point bend results demonstrate utility in component-scale simulations}

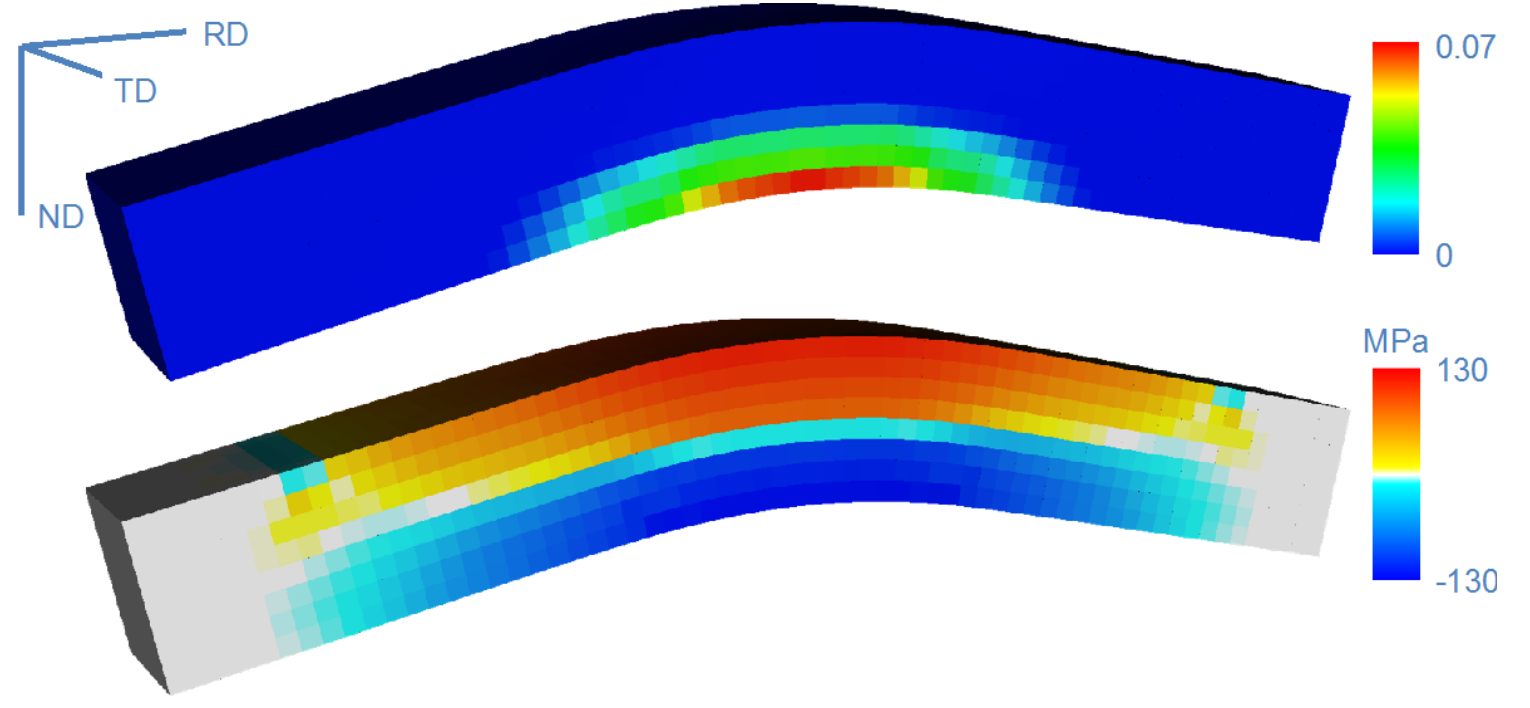

Twin fraction: tensioncompression asymmetry

Shape of cross-sections for two different bend orientations - agree with published results (Knezevic et al., Mat. Sci. Eng. A, 2013)
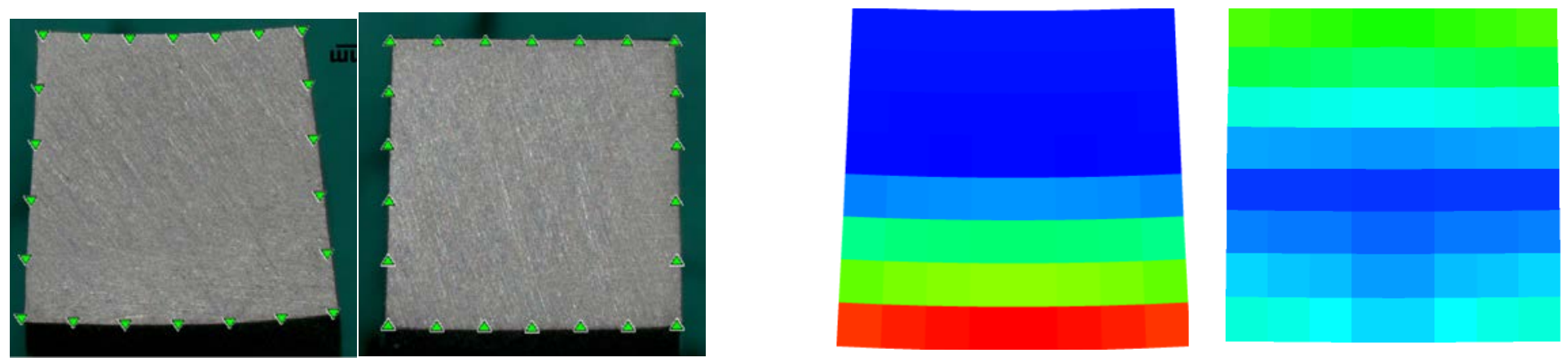


\section{Summary}

- Adaptive sampling working with more detailed state descriptions:

- ODF expansion with 10s of terms

- Maintains benefit from sampling

- Convergence versus expansion order is well behaved

- Gross texture evolution from twinning is captured

- VPSC derivatives: in parallel on remote processes

- AS-VPSC improves AS-Taylor accounting for texture evolution,

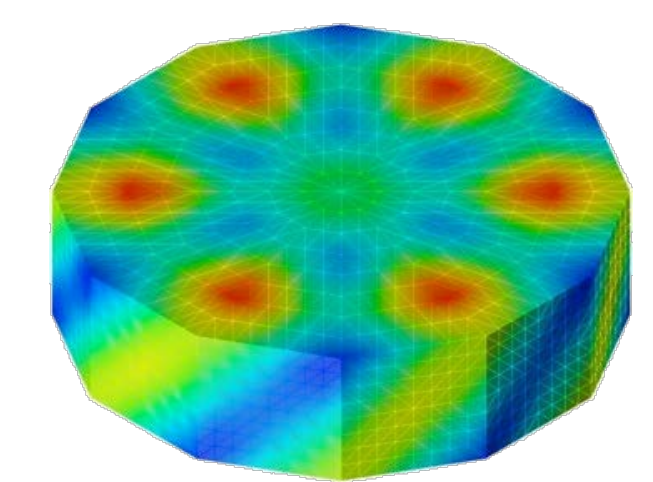
twinning effects.

- ALE3D-AS-VPSC (this work) vs COEVP-AS-VPFFT (EXMatEx scale-bridging app):

- homogenization-based model (VPSC) implies no fine-scale spatial resolution, allowing us to account for microstructure evolution by solving a PDE (flux equation with source and sinks) for ODF.

- with VPFFT we still need to find appropriate and numerically tractable microstructure representation for AS.

- Do we want to pursue a COEVP-AS-VPSC scale-bridging app? 